\title{
ITUR 2 - DIMENSIONAMENTO DAS REDES DE CABOS COAXIAIS
}

\section{Introdução}

No último século, o sector das comunicações passou de um estado inicial de simples curiosidade tecnológica até um dos mais dinâmicos pilares económicos de vários países por todo o mundo.

A procura por mais e melhores serviços de televisão e internet levaram à necessidade de evoluir as tecnologias existentes de modo a conseguir cumprir com a elevada procura ao mesmo tempo que apresentam um serviço mais rápido, mais fiável e de melhor qualidade.

A União Europeia (EU) colocou como objetivo a conversão de todo o seu território de radiodifusão analógica para a tecnologia digital. Deste modo, não só se conseguiu uma mais eficiente utilização do espetro radioelétrico, como se conseguiu aumentar o número de canais a transmitir, sendo estes de melhor qualidade que a tecnologia analógica, pois deu a possibilidade de várias emissoras poderem emitir em High Definition (HD).

Não foi apenas na televisão que se deram grandes avanços, mas também na internet. Nos últimos anos foi verificado um grande aumento nas velocidades atingidas por estas ligações, sendo que hoje, a maior parte das ligações são incorporadas com serviços de televisão.

Em Portugal também se verificou uma grande prosperidade para o dia-a-dia das populações e empresas, como pode ser verificado na Figura 1, que compara a evolução do PIB com a evolução do número de subscritores de diferentes serviços de telecomunicações tais como telefone fixo, móvel, internet e televisão paga.

Para levar estas tecnologias aos lares da população com a mais alta qualidade de serviço, são necessárias várias infraestruturas, sendo as ITUR (infra estruturas de telecomunicações em urbanizações, loteamentos e conjunto de edifícios) parte integrante dessas infraestruturas tornando-se assim a sua qualidade e regulação pelas normas de extrema importância, sendo que estas são hoje em dia regulamentadas pelo Manual de ITUR 2a Edição, que é no fundo um conjunto de normas e diretrizes a seguir no projeto, dimensionamento e execução deste tipo de infraestruturas de modo a poder levar à casa dos utilizadores.

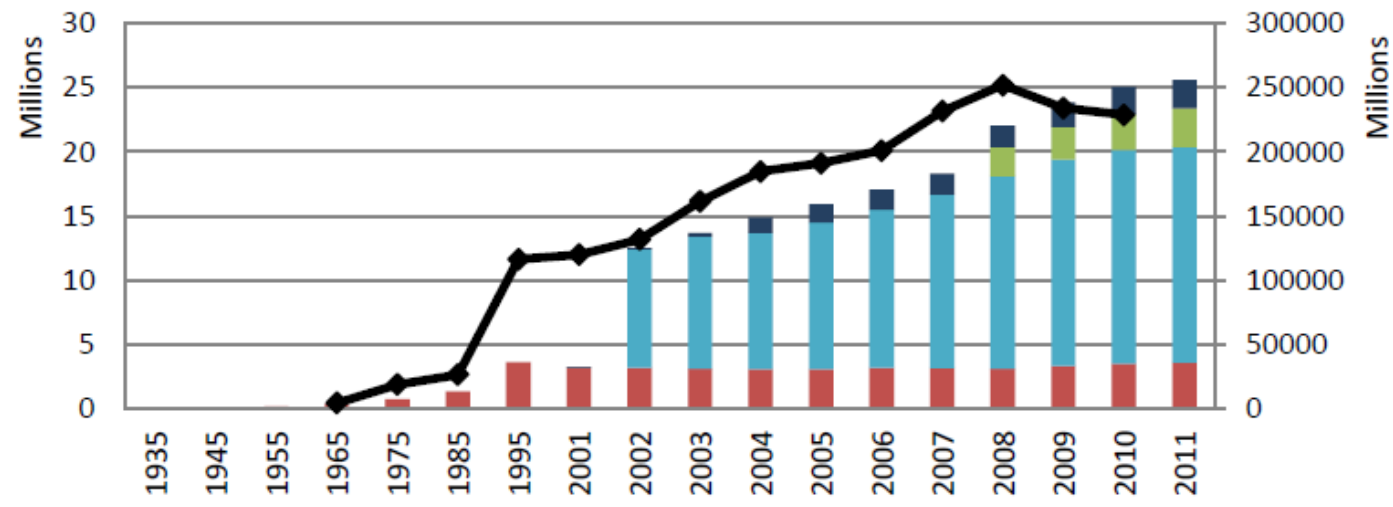

Fixed telephone $\quad$ Mobile telephone
Fixed internet $\quad$ GDP Portugal (right axis)

Figura 1. Evolução da penetração de telecomunicações (eixo da esquerda) versus evolução do PIB em Portugal (eixo da direita) de 1965 a 2011 [Fonte: ANACOM, INE e World Bank] 
Uma ITUR Privada tem que prever o alojamento das 3 tecnologias previstas pelo manual de ITUR em vigor, o Par de Cobre, a Fibra Ótica e o cabo Coaxial, contudo neste artigo vamo-nos focar apenas na tecnologia de cabo Coaxial, que apesar de ser uma tecnologia com alguns anos, tem ainda os seus pontos fortes. Uma grande vantagem desta tecnologia é a imunidade a interferências externas aliadas ao seu baixo custo o que faz dele uma tecnologia ainda muito usada apesar de ser o que apresenta maiores atenuações por unidade de comprimento.

\section{Surgimento das ITUR}

O desenvolvimento das atividades económicas e sociais, os enormes progressos tecnológicos, verificados e as novas exigências decorrentes do ambiente concorrencial estabelecido em Portugal, impuseram a necessidade de formular novas regras técnicas para o projeto, instalação e gestão das Infraestruturas de Telecomunicações, com suporte as tecnologias de cabo de pares de cobre, cabo coaxial e fibra ótica.

Com a publicação do Decreto-Lei n.ำ123/2009, de 21 de maio, iniciou-se um conjunto de alterações em todo o enquadramento de atividade relativa às infraestruturas de telecomunicações em edifícios, as chamadas ITED, assim como nas infraestruturas de telecomunicações em Loteamentos, Urbanizações e Conjunto de Edifícios.

Alguns dos aspetos que a nova legislação introduziu foram:

- Inscrição dos técnicos de projeto e de instalação nas associações profissionais e a necessidade de formação;

- A criação do ITUR (Infra estruturas de telecomunicações em urbanizações e conjunto de edifícios);

- Necessidade de novos regulamentos;

- Acrescidas responsabilidades para os projetistas e instaladores assim como para os donos de obra.
O manual de ITUR - 1a Edição foi aprovado por deliberação CA, da ANACOM, de 25 de Novembro de 2009 sendo que a sua entrada em vigor foi a 1 de Janeiro de 2010.

O regime jurídico aplicável às Infraestruturas de Telecomunicações em Loteamentos, Urbanizações e Conjunto de edifícios, consagra a obrigatoriedade de construção das ITUR em duas realidades distintas, ITUR Públicas e ITUR Privadas.

As ITUR Públicas exigem o dimensionamento da rede de tubagens ou tubagem para instalação de cabos, equipamentos e outros dispositivos, incluindo armários de telecomunicações, caixas e câmaras de visita. São integrantes do domínio público municipal, o proprietário e titulares dos direitos reiais cedem gratuitamente ao município as ITUR instaladas, sendo que a sua gestão e conservação cabem aos municípios.

As ITUR Privadas, além do exigido pelas ITUR Públicas, exigem ainda a cablagem em par de cobre, cabo coaxial e fibra ótica para ligação às redes públicas de comunicações eletrónicas, bem como instalações elétricas de suporte a equipamentos e sistemas de terra. São de propriedade privada e a sua gestão é feita pela administração.

\section{- ITUR 2a Edição}

A 20 de Novembro de 2014 foi aprovada por decisão da ANACOM a 2a edição do Manual de ITUR - Prescrições e especificações técnicas das infraestruturas de telecomunicações em loteamentos, urbanizações e conjuntos de edifícios.

Esta 2.a edição do Manual ITUR resulta de alterações introduzidas no Manual ITED (3.a edição), bem como da normal evolução normativa europeia, que consequentemente conduziram à necessidade de adaptação das regras técnicas aplicáveis às infraestruturas exteriores aos edifícios, nomeadamente as existentes em loteamentos, urbanizações e conjuntos de edifícios e, assim, à revisão do Manual ITUR. 
- Partes constituintes de uma rede coaxial de uma ITUR Privada

Uma ITUR privada pode ter origem na rede pública ou numa ITUR Pública, sendo que o seu ponto de fronteira em ambos os casos é dado no ATU. O final de uma ITUR privada é dado nos primários dos repartidores gerais (RG) no caso de edifícios e nos primários dos repartidores de cliente (RC) no caso de moradias unifamiliares.

Para uma rede de cabos coaxiais o manual de ITUR 2a Edição obriga à garantia de que em cada ATE, ou ATI, exista a possibilidade de ligação, como mínimo, de uma rede de distribuição de CATV. O projetista pode considerar a existência de uma outra rede, para distribuição do sistema de S/MATV.

Uma rede de cabos coaxial duma ITUR privada terá início no secundário do repartidor de urbanização de cabo coaxial (RU-CC) e finda no primário do repartidor geral de cabo coaxial de um edifício coletivo (RG-CC) ou repartidor de cliente de cabo coaxial (RC-CC) no caso de se tratar de um único fogo, local onde terá inicio a rede ITED.

São parte integrante da rede de cablagem coaxial os cabos da rede principal, os cabos de distribuição, os conectores, repartidores, derivadores e amplificadores.

O cabo da rede principal é utilizado na ligação entre amplificadores e na ligação destes a outros dispositivos, nomeadamente derivadores e repartidores. O cabo da rede de distribuição é destinado à ligação aos repartidores gerais (RG-CC) e repartidores de cliente (RG-CC), fazendo a ligação destes à rede principal.

\section{- Projeto da rede}

Deve ser prevista no mínimo uma rede de coaxial CATV que, dependendo da dimensão da ITUR, pode ser híbrida (coaxial e fibra), devendo ser desenvolvida a partir do ATU, servindo todos os fogos e o seu dimensionamento deve considerar e ter em conta os limites previstos para a distribuição de sinais de TV e de dados (DOCSIS).
O projetista para as redes de coaxial deve adotar as soluções que melhor se adaptem às necessidades da ITUR, podendo projetar as seguintes topologias:

- Estrela - chegada ao ATE, ou ATI, de um cabo coaxial de classe TDC-C-H proveniente do ATU por cada fogo (aplicável essencialmente em ITUR de reduzida dimensão), embora seja a mais adequada pois permite a ligação de vários operadores em simultâneo.

- Árvore - chegada ao ATE, ou ATI, de um cabo coaxial de classe TDC-C-H para repartição (no primário do RG ou RC) por todos os fogos;

- Mista - combinação das topologias anteriores.

A rede de cabos coaxiais pode ser dividida em dois troços distintos:

- Rede principal - troço limitado a montante pelo ATU e a jusante pelos amplificadores de distribuição. Dependendo da dimensão da ITUR e das condições referidas anteriormente, este troço pode ser suportado por uma ligação em fibra ótica;

- Rede de distribuição - troço limitado a montante pela rede principal sendo destinado à ligação da rede principal aos repartidores gerais (RG-CC) e repartidores de cliente (RGCC).

Poderá ainda ser considerado o transporte e distribuição de sinais S/MATV (sinais do tipo B - via satélite) e sinais MATV (tipo A - via hertziana terrestre) na mesma rede, caso se garanta a necessária compatibilidade de espectro, ou ser efetuado em redes independentes.

\section{Aplicação informática desenvolvida}

Os requisitos previstos para uma infraestrutura de cabo coaxial pelo manual de ITUR são sem dúvida os mais exigentes, deste modo foi desenvolvida uma ferramenta de cálculo destinada ao auxílio do dimensionamento das redes de cabos coaxiais das ITUR Privadas. 
Como foi visto anteriormente uma rede de cabos coaxiais numa ITUR Privada é composta por certos equipamentos chave, tais como o ATU de onde sairão todas as cablagens a ser distribuídas, os próprios cabos e seus conectores, sejam da rede principal ou da rede de distribuição, repartidores e derivadores e finalmente, se necessário, amplificadores.

A ferramenta foi desenvolvida de modo a ter em conta todos estes componentes e suas características, tendo para isso sido utilizados dados referentes a duas marcas presentes no mercado das telecomunicações, a TEKA e a TELEVES.

\section{- Modo de Funcionamento}

Assim que se abre o ficheiro Excel a única informação que pode ser vista é o cabeçalho (Figura 2) do que virá a ser a nossa tabela.

Tudo foi desenvolvido de modo a aumentar a simplicidade e facilidade de uso ao seu utilizador, contudo, é necessário possuir conhecimentos de ITUR para a correta utilização da ferramenta. Antes de mais é necessário termos previamente um rascunho de como estará distribuída a nossa rede de cablagem, por exemplo, quantos derivadores ou repartidores vamos ter, quantos lotes iremos alimentar, etc.

Para exemplificar o funcionamento da ferramenta vamos dimensionar a seguinte rede, bastante simples com apenas 1 saída do ATU e dois derivadores, que vai alimentar 6 lotes de moradias unifamiliares.

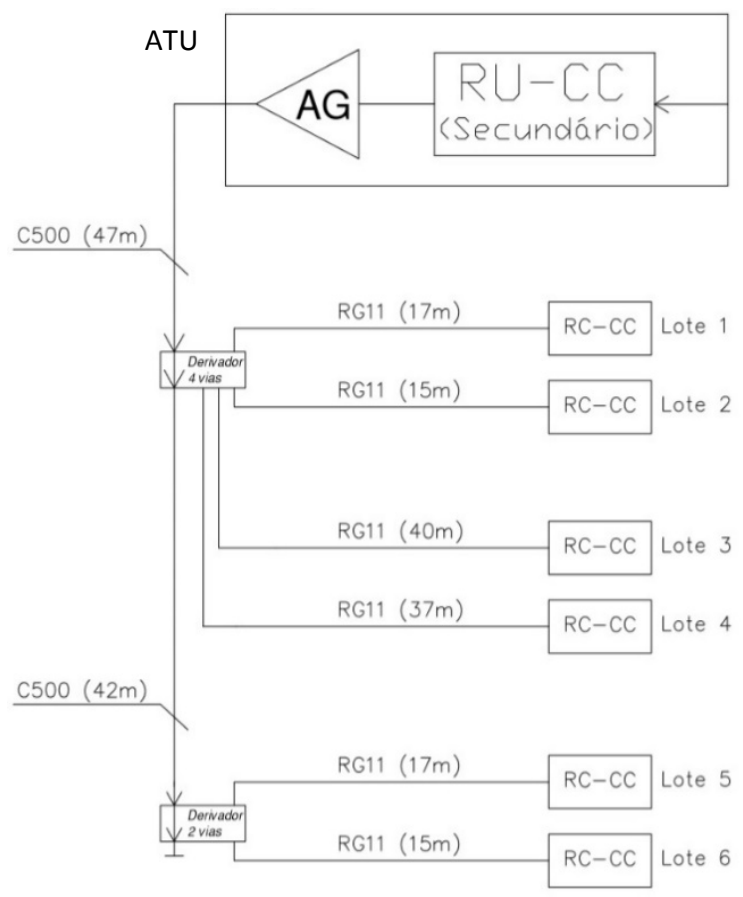

Figura 3. Exemplo de uma arquitetura de rede ITUR

Para iniciarmos o desenvolvimento da rede, deve ser selecionada a opção "Adicionar Saída ATU" seguindo posteriormente as instruções para definir toda a rede de uma vez. Ao selecionar esta opção deve certificar-se que não comete erros, uma vez que esta ação é irreversível, ou seja, uma vez acionada não é possível voltar atrás com a mesma, sendo para isso necessário fazer reset a toda a rede já definida. Assim sendo, a primeira coisa que a ferramenta faz é adicionar uma linha à tabela que será a primeira saída do ATU.

\section{Adicionar Saída ATU}

\begin{tabular}{|c|c|c|c|c|c|c|c|c|c|c|c|c|c|c|}
\hline \multicolumn{15}{|c|}{ Dimensionamento da rede de cabo Coaxial } \\
\hline \multirow{2}{*}{$\begin{array}{c}\text { Saida } \\
\text { ATU }\end{array}$} & \multirow{2}{*}{ Inicio } & \multirow{2}{*}{\multicolumn{2}{|c|}{ Fim }} & \multirow{2}{*}{$\left|\begin{array}{c|}\text { Comp. } \\
\text { cabo (m) }\end{array}\right|$} & \multirow{2}{*}{ Cabo } & \multirow{2}{*}{\begin{tabular}{|c|} 
Atenuação total \\
do cabo (dB)
\end{tabular}} & \multicolumn{2}{|c|}{ Derivador/Repartidor } & & Amplificador & \multirow{2}{*}{ SNR } & \multirow{2}{*}{$\begin{array}{c}\text { Atenuação Total } \\
\text { do Troço (dB) }\end{array}$} & \multirow{2}{*}{ Tilt } & \multirow{2}{*}{ Sinal (dB) } \\
\hline & & & Tipo & & & & Referência & Perdas & Ref. & \begin{tabular}{l|l}
\multicolumn{2}{c|}{ Ganho } \\
$47 \mathrm{MHz}$ & $862 \mathrm{MHz}$
\end{tabular} & & & & \\
\hline
\end{tabular}

Figura 2. Formatação inicial das funcionalidades da aplicação informática proposta

\begin{tabular}{|c|c|c|c|c|c|c|c|c|c|c|c|c|c|c|c|}
\hline \multicolumn{16}{|c|}{ Dimensionamento da rede de cabo Coaxial } \\
\hline Saída & \multirow{2}{*}{ Inicio } & \multirow{2}{*}{\multicolumn{2}{|c|}{ Fim }} & \multirow{3}{*}{$\left|\begin{array}{c}\text { Comp. } \\
\text { cabo (m) }\end{array}\right|$} & \multirow{2}{*}{ Cabo } & \multirow{2}{*}{\begin{tabular}{|c|} 
Atenuação total \\
do cabo (dB)
\end{tabular}} & \multirow{2}{*}{\multicolumn{2}{|c|}{ Derivador/Repartidor }} & \multirow{2}{*}{\multicolumn{3}{|c|}{ Amplificador }} & \multirow{3}{*}{ SNR } & \multirow{2}{*}{$\begin{array}{c}\text { Atenuação Total } \\
\text { do Troço (dB) }\end{array}$} & \multirow{2}{*}{ Tilt } & \multirow{3}{*}{$\begin{array}{l}\text { Sinal (dB) } \\
862 \mathrm{MHz}\end{array}$} \\
\hline ATU & & & & & & & & & & & & & & & \\
\hline & & Descrição & Tipo & & & \begin{tabular}{l|l|l}
$47 \mathrm{MHz}$ & $862 \mathrm{MHz}$ \\
\end{tabular} & Referência & Perdas & Ref. & $47 \mathrm{MHz}$ & $862 \mathrm{MHz}$ & & \begin{tabular}{l|l|l}
$47 \mathrm{MHz}$ & $862 \mathrm{MHz}$ \\
\end{tabular} & & \\
\hline$\overline{11}$ & ATU & & & & $\overline{c \text { C500 Teka }}$ & 0 & & & $\mathrm{~N} / \mathrm{A}$ & & & & 1 & & 0 \\
\hline
\end{tabular}

Figura 4. Saída 1 do ATU selecionada 
Seguidamente deve ser escolhido o destino da primeira saída do ATU, se um repartidor ou um derivador. Um repartidor deve ser apenas usado no caso de ser necessário repartir a rede, seguindo a rede principal dois caminhos distintos. Em todos as restantes situações devem ser utilizados derivadores.

Neste caso será escolhido um derivador, sendo logo de seguida feita a escolha do número de saídas pretendidas. As soluções previstas permitem derivadores de 2 e 4 vias, ou seja, 4 vias de derivação (Entrada-Derivação) e uma via de inserção.

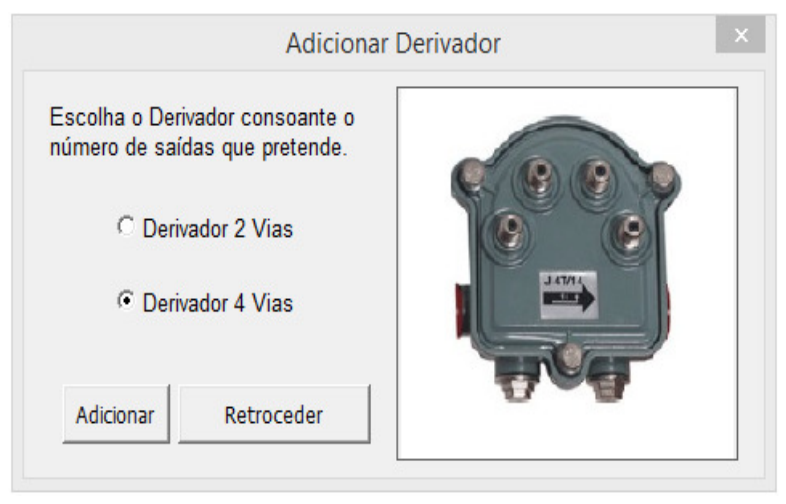

Figura 5. Exemplo da funcionalidade "Adicionar Derivador"

Logo de seguida o utilizador é proposto com várias opções, podendo adicionar outro derivador em série com o anterior, fazer uma repartição, criar uma nova saída do ATU ou terminar a rede.
Se inicialmente se adicionar um derivador de 4 vias, seguido de um derivador de 2 vias, surge a opção terminar rede, resultando daí a seguinte tabela.

A partir deste momento tudo o que o utilizador necessita de fazer é introduzir o comprimento dos cabos e todas as atenuações serão imediatamente calculadas dando como resultado o nível de sinal no local de chegada na última coluna. Por motivos de organização e melhor perceção o utilizador deverá escrever na coluna "Descrição" o local onde termina a cablagem, contudo, não é obrigatório.

A coluna "Tipo" é composta por uma "Drop-Down List" onde se pode escolher o tipo de chegada, se um Edifico, se uma Moradia unifamiliar ou "N/A" no caso da saída do derivador não estar atribuída.

\begin{tabular}{|c|c|c|c|c|}
\hline \multirow{2}{*}{\multicolumn{2}{|c|}{ Inicio }} & \multicolumn{2}{|c|}{ Fim } & \\
\hline & & Descrição & Tipo & \\
\hline \multicolumn{2}{|c|}{ ATU } & D1 & & \\
\hline \multirow{5}{*}{ D1 } & Saída 1 & & Moradia & \\
\hline & Saída 2 & & Moradia & I \\
\hline & Saída 3 & & \multirow{2}{*}{\begin{tabular}{|l} 
Moradia \\
Edifico \\
N/A \\
\end{tabular}} & \\
\hline & Saída 4 & & & \\
\hline & Inserção & D2 & & \\
\hline \multirow{3}{*}{ D2 } & Saída 1 & & Edifico & \\
\hline & Saída 2 & & Edifico & \\
\hline & Inserção & & & \\
\hline
\end{tabular}

Figura 6. Exemplo do tipo de chegada

A coluna "Cabo" também é composta por uma Drop-Down List onde se pode escolher o tipo de cabo que se quer utilizar.

\begin{tabular}{|c|c|c|c|c|c|c|c|c|c|c|c|c|c|c|c|c|c|c|}
\hline \multicolumn{19}{|c|}{ Dimensionamento da rede de cabo Coaxial } \\
\hline \multirow{3}{*}{\begin{tabular}{|c|} 
Saida \\
ATU
\end{tabular}} & & \multirow{2}{*}{\multicolumn{2}{|c|}{ Fim }} & \multirow{3}{*}{$\begin{array}{c}\text { Comp. } \\
\text { cabo (m) }\end{array}$} & \multirow{3}{*}{ Cabo } & \multirow{2}{*}{\multicolumn{2}{|c|}{\begin{tabular}{|c|} 
Atenuação total \\
do cabo (dB)
\end{tabular}}} & \multirow{2}{*}{\multicolumn{2}{|c|}{ Derivador/Repartidor }} & \multirow{2}{*}{\multicolumn{3}{|c|}{ Amplificador }} & \multirow{3}{*}{ SNR } & \multirow{2}{*}{\multicolumn{2}{|c|}{\begin{tabular}{|c} 
Atenuação Total \\
do Troço (dB)
\end{tabular}}} & \multirow{3}{*}{ Tilt } & \multirow{3}{*}{\begin{tabular}{|l} 
Sinal (dB) \\
$862 \mathrm{MHz}$
\end{tabular}} \\
\hline & & & & & & & & & & & & & & & & & & \\
\hline & \multicolumn{2}{|c|}{ Inicio } & Descrição & Tіро & & & $47 \mathrm{MHz}$ & $862 \mathrm{MHz}$ & Referência & Perdas & Ref. & $47 \mathrm{MHz}$ & $862 \mathrm{MHz}$ & & \begin{tabular}{|l|l}
$47 \mathrm{MHz}$ \\
\end{tabular} & $862 \mathrm{MHz}$ & & \\
\hline 1 & \multicolumn{2}{|c|}{$\overline{\overline{\text { ATU }}}$} & D1 & & & C500 Teka & 0 & 0 & & & $\mathrm{~N} / \mathrm{A}$ & & & & 1 & 1 & & 70 \\
\hline & \multirow{5}{*}{ D1 } & Saída 1 & & Moradia & & RG11 Teka & 0,00 & 0,00 & \multirow{5}{*}{$\begin{array}{c}\text { Teka [J-4T/11] } \\
11 \mathrm{~dB}\end{array}$} & 11 & & & & & 13,00 & 13,00 & 0,00 & 57,00 \\
\hline & & \begin{tabular}{|l|} 
Saida 2 \\
\end{tabular} & & Moradia & & RG11 Teka & 0,00 & 0,00 & & 11 & & & & & 13,00 & 13,00 & 0,00 & 57,00 \\
\hline & & \begin{tabular}{|l|} 
Saida 3 \\
\end{tabular} & & Moradia & & RG11 Teka & 0,00 & 0,00 & & 11 & & & & & 13,00 & 13,00 & 0,00 & 57,00 \\
\hline & & Saída 4 & & Moradia & & RG11 Teka & 0,00 & 0,00 & & 11 & & & & & 13,00 & 13,00 & 0,00 & 57,00 \\
\hline & & \begin{tabular}{|l|} 
Inserção \\
\end{tabular} & D2 & & & C500_TEKA & 0 & 0 & & 4,8 & $\mathrm{~N} / \mathrm{A}$ & & & & 6,80 & 6,80 & & 63,20 \\
\hline & \multirow{3}{*}{ D2 } & Saída 1 & & Moradia & & RG11 Teka & 0 & 0 & \multirow{3}{*}{$\begin{array}{c}\text { Teka [J-2T/8] } \\
8 \mathrm{~dB}\end{array}$} & 8 & & & & & 15,80 & 15,80 & 0,00 & 54,20 \\
\hline & & \begin{tabular}{|l|} 
Saída 2 \\
\end{tabular} & & Moradia & & RG11 Teka & 0 & 0 & & 8 & & & & & 15,80 & 15,80 & 0,00 & 54,20 \\
\hline & & \begin{tabular}{|l|} 
Inserção \\
\end{tabular} & & & & C500 Teka & 0 & 0 & & 5,2 & $\mathrm{~N} / \mathrm{A}$ & & & & 13,00 & 13,00 & & 57,00 \\
\hline & & & & & & & & & & Relação & Sinal $\mathrm{F}$ & uido total & da saída & & & & & \\
\hline
\end{tabular}

Figura 7. Exemplo do dimensionamento da rede de cabos coaxial definida 
O mesmo acontece na coluna "Derivador/Repartidor" onde se pode escolher um dos vários modelos disponíveis de acordo com a atenuação pretendida e com o seu fabricante tendo a possibilidade de utilizar derivadores da TEKA e da TELEVES

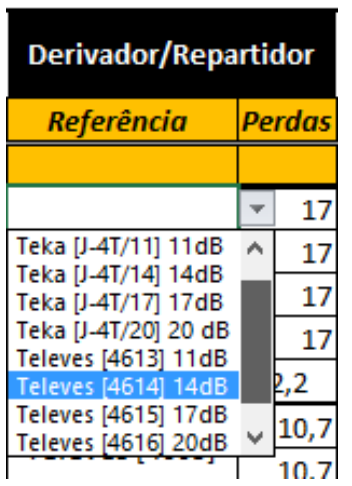

Figura 8. Seleção da atenuação e fabricante do derivador

Também é possivel escolher um ganho de amplificação que afetará todos os componentes que estejam a jusante desse ponto. Apenas está prevista a utilização de amplificadores na linha principal.

A Figura 9 iliustra um exemplo de uma rede definida, onde se pode ver as condições de sinal à chegada assim como o valor de Tilt e SNR.

Caso as condições previstas no ITUR não sejam cumpridas a célula em questão ficará vermelha, o que indica que o utilizador terá que jogar com o valor das atenuações dos derivadores e com o ganho dos amplificadores de modo a cumprir todos os requisitos.
Podem ser adicionadas tantas saídas do ATU quantas forem necessárias, sendo que estas serão totalmente independentes umas das outras, à exceção do sinal de entrada do ATU que é definido no botão "Sinal entrada ATU" no topo da página, e esse será o sinal que alimenta o Repartidor de Urbanização de Cabo Coaxial (RU-CC).

Ao criar uma nova saída do ATU é necessário que se definia todos os componentes dessa saída. Assim como no caso de se adicionarem repartidores, será pedido ao utilizador para definir todos os componentes que ligarão a cada uma das saída visto não ser possível adicionar mais componentes posteriormente.

\section{Conclusões}

Neste trabalho foi proposto o desenvolvimento de uma ferramenta de cálculo destinada ao auxílio do dimensionamento de redes de cabo coaxial nas ITUR Privadas prevendo para o efeito soluções técnicas de dois fabricantes distintos, sendo para isso necessário efetuar uma pesquisa de equipamentos de redes de cabo coaxial disponíveis no mercado.

A aplicação foi desenvolvida com recurso ao software Microsoft Excel tomando partido das suas avançadas funções possibilitadas pelo uso do Visual Basic for Applications.

Esta ferramenta poderá constituir uma ajuda aos projetistas para dimensionamento das redes coaxiais das ITUR privadas.

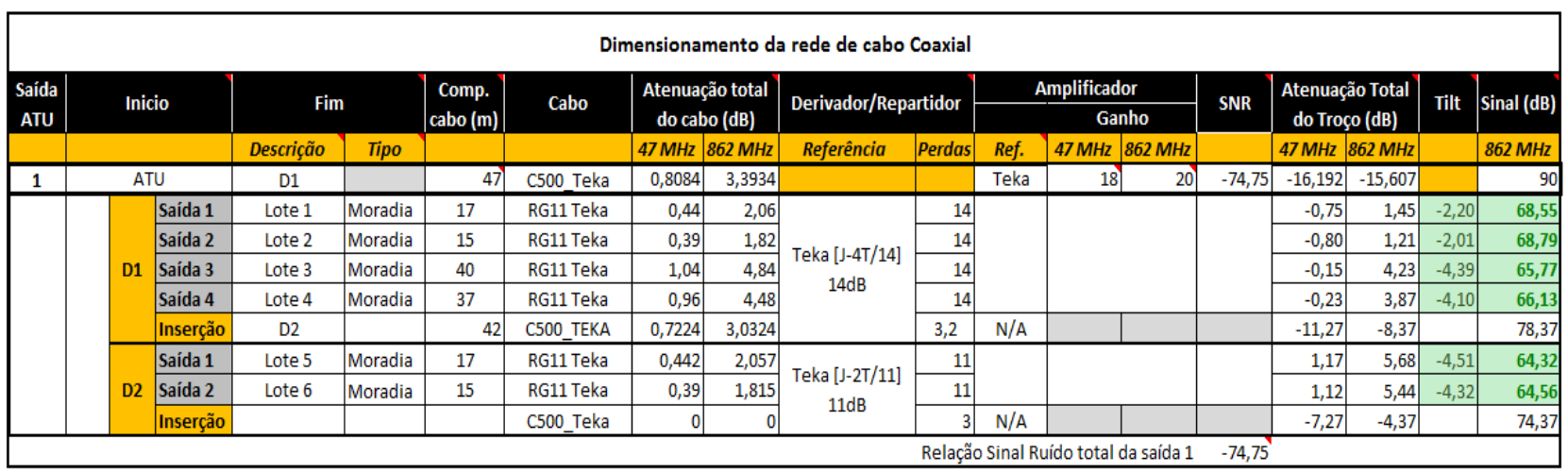

Figura 9. Tabela preenchida onde se pode ver as condições de Nível de Sinal e Tilt 